\title{
AVALIAÇÃO PSICOLÓGICA DOS EFEITOS SECUNDÁRIOS DA TRIAGEM SISTEMÁTICA DA SURDEZ NEONATAL PERMANENTE
}

\section{PSYCHOLOGICAL EVALUATION OF THE SECONDARY EFFECTS OF NEONATAL HEARING SCREENING}

\author{
Barbara Le Driant* \\ Luc Vandromme* \\ Catherine Kolski* \\ Valdimir Strunski**
}

Driant bl, vendrome 1, kolski c, strunski v Avaliação psicológica dos efeitos secundários da triagem sistemática da surdez neonatal permanente. Rev Bras Cresc Desenv Hum 2004; 14(2): 31-34;

Resumo: O objetivo deste estudo é avaliar o impacto psicológico da triagem sistemática da surdez nos pais das crianças criadas. Informar os pais de uma surdez potencial desde os primeiros d^las de vida pode provocar uma angústia materna assim como uma desorganização das primeiras relações mae-criança. Os resultados obtidas mostram que os pais apresentam um nível alto de ansiedades porém mantendo interações de qualidade com o seu bebê.

Palavras-chave: Surdez neonatal. Triagem. Ansiedade parental. Interações precoces.

\section{INTRODUÇÃO}

A triagem da surdez neonatal permanente (SPN), definida por uma perda total permanente superior ou igual a $40 \mathrm{dBHL}$ na orelha melhor, inscreve-se em um processo de prevenção secundária dos handicaps. Como sublinha o relatório da ANAES I, a surdez infantil é um problema de saúde pública. Handicap grave e freqüente (1,2 a 3,1 por 1000 nascimentos segundo os estudos), a SPN implica em uma privação sensorial, que tem conseqüências não apenas no desenvolvimento da linguagem mas, de uma maneira mais geral, no desenvolvimento cognitivo da criança. Assim, a freqüência, as conseqüências da privação auditiva no desenvolvimento da criança, tanto quanto o interesse nos cuidados precoces ${ }^{2-4}$, justificam uma triagem sistemática da surdez na criança no período neonatal.

Contudo, informar aos pais uma disfunção sensorial potencial desde os primeiros dias de vida da criança pode provocar uma certa angústia parental assim como uma desorganização das primeiras relações pais-criança, e certos profissionais se posicionam de modo reservado quanto aos reais benefícios de uma triagem praticada durante o período neonatal, que se revela sensível em inúmeros aspectos ${ }^{5}$.

Vários estudos tentaram avaliar as conseqüências de um resultado positivo no teste de triagem da surdez permanente neonatal e emitem conclusões bastante contrastantes. Um certo número deles relatam um aumento da ansiedade parental quando a criança foi triada positiva no primeiro teste ${ }^{6}$ e insistem sobre a importância da realização de um acompanhamento durante o período que separa o primeiro do segundo teste ${ }^{7}$. Estes estudos relatam que o tempo de duração entre os dois testes tem um papel importante. Com efeito, mesmo se o nível de ansiedade medida permanece baixo, os pais confessam estar preocupados com o teste de triagem e estar particular-

\footnotetext{
Equipe ECCHAT - Laboratoire d’Efficience Cognitive dans les Concuites Humaines d’Apprentissage et de Travail, Université de Picacardie Jules Verne, Chemin du Thil, 80000 Amiens - courriel: barbara.le-driant@ca.u-picardie.fr

** Serviço ORL - Otorrinolaringologia e Cirurgia Cérvico-facial do CHU - Centro Hospitalar Universitário, de Amiens.
} 
mente atentos à maneira como a sua criança reage aos diferentes barulhos do entorno ${ }^{8}$. Uma informação de qualidade sobre os objetivos e os benfícios da triagem da surdez neonatal parece contribuir a uma diminuição da ansiedades. Outros estudos não concluem quanto à existência de efeitos secundários da triagem nem sobre a ansiedade materna nem sobre a qualidade da interação mãe-criança. Contudo, se bem que não se nota um aumento significativo da ansiedade, cada um destes estudos indica alguns pais que confessam estar muito preocupados com as capacidades auditivas de seus filhos.

Deve-se notar que os diferentes estudos citados utilizam, na maior parte das vezes, metodologias baseadas seja em questionários enviados pelo correio seja em entrevistas realizadas por meio do telefone e pode-se colocar a questão da sensibilidade de tais instrumentos de avaliação.

Deste modo, o trabalho que apresentamos aqui visa avaliar o impacto de um programa de triagem da SPN na ansiedade parental e na qualidade das interações pais-criança. Ele foi realizado em colaboração com o serviço de ORL Otorrino-Laringologia e de Cimgia Cérvicofacial dirigida pelo Prof. Strunski (CHU - Centre Hospi-talier Universitaire, de Amiens, Picardie, França).

\section{MÉTODOS}

\section{População}

A população estudada consistiu em 80 díades mãe-criança, 60 participaram dc programa de triagem e 20 não participaram da triagem e são utilizadas como grupo controle.

Cada uma das crianças participantes do programa de triagem realiza, durante a sua permanência na maternidade, um teste de triagem de surdez neonatal permanente por meio de um registro das otoemissões acústicas provocadas (OEAP) nas duas Grelhas. O teste é dito negativo quando as OEAP estão presentes nos dois lados, unilateral positivo se as OEAP são registradas em uma única orelha e positivo bilateral se as OEAP estão ausentes nas duas Grelhas. No grupo triado, 20 crianças obtiveram um resultado negativo no teste, 20 um resultado positivo unilateral e 20 um resultado positivo bilateral.

As crianças triadas positivas devem refazer um segundo teste de triagem 8 semanas após. A informação referente ao procedimento, aos objetivos e aos benefícios de uma tal triagem é fornecida aos pais pelo pessoal encarregado na maternidade, e isto antes de que o teste de triagem seja realizado.

\section{Procedimento}

$\mathrm{O}(\mathrm{s})$ psicólogo(s) realiza(m) duas entrevistas semi-diretivas com cada uma das mães cujo filho será objeto de uma triagem da SPN. A primeira entrevista é realizada depois do anúncio do resultado positivo ou negativo do teste de triagem e a segunda entrevista se efetua antes do segundo teste de triagem que acontece 8 semanas mais tarde.

Afim de avaliar o nível de ansiedade materna assim como a qualidade da relação mãecriança, a psicóloga se apoia nos resultados de várias escalas cuja sensibilidade foi avaliada por um estudo precedente, realizado com díades mãe-criança cuja criança não era objeto de um programa de triagem. No que se refere à avaliação da anisiedade, estas escalas são a MADRS (Montgomery, Asberg Depression Rating Scale, 1979), certos itens do questionnário EPDS (Edinburgh postnatal scale depression, J. Cox, 1987) assim como certos itens do autoquestionário de Kennerley (1987). A avaliação das interações se baseia em uma escala de interação.

\section{RESULTADOS}

De um modo geral, a triagem gera ansiedade nas mães $(F(2)=6,99 ; p=0,003)$. Este aumento da ansiedade materna está ligado ao resultado positivo do teste dado que não se observa diferença significativa entre as mães do grupo controle e as mães do grupo de crianças triadas negativas $(\mathrm{t}(38)=0,31$; ns).

Por outro lado, no caso de um resultado positivo, seja ele unilateral $(\mathrm{t}(38)=5,82$; $\mathrm{p}=0,00001)$ ou bilatéral $(\mathrm{t}(38)=5,11$; $\mathrm{p}=0,000009$ ), observa-se um aumento significativo da ansiedade materna (Cf. Gráfico 1).

A qualidade das interações não se altera com a triagem. Contudo, no caso de um resultado positivo bilateral ao teste de triagem, as interações são de uma qualidade pior do que quando o resultado se revela negativo $(\mathrm{t}(38)=2,61$, $\mathrm{p}=0,01)$.

A ansiedade das mães cuja criança obteve um resultado positivo no teste de triagem não evolui entre a primeira e a segunda entrevista, seja ter sido o resultado unilateral $(\mathrm{t}(38)=1,83$; n.s $)$ ou bilateral (t(38) = 0,767; n.s). Ocorre o mesmo para o estado psíquico das mães, que permanece estável entre as duas entrevistas. (Resultado unilateral: $\mathrm{t}(38)=1,019$, n.s; Resultado bilateral: $\mathrm{t}(38)=0,767, \mathrm{n} \cdot \mathrm{s})$. As interações permanecem com uma boa qualidade, e isto para todos os grupos considerados. Não se observam mais diferenças entre os grupos segundo o resultado do teste, na segunda entrevista. 


\section{DISCUSSÃO}

O presente estudo tinha por objetivo avaliar os efeitos secundários de uma triagem sistemática da surdez neonatal. Os resultados se distinguem claramente dos estudos precedentes que concluíam haver uma ausência de ansiedade parental ligada à realização do teste de triagem 89. Com efeito, se bem que o teste em si mesmo não pareça ter consequências sobre o estado psicológico da mãe, um resultado positivo neste último acarreta um aumento importante da ansiedade materna que altera o seu estado psicológico geral, e tanto mais quanto o teste de triagem se revela positivo nas duas Grelhas. Apesar da alta ansiedade levantada nas mães, as interações observadas entre os dois tudo precedente, realizado com díades mãe-criança cuja criança não era objeto de um programa de triagem. No que se refere à avaliação da anisiedade, estas escalas são a MADRS (Montgomery, Asberg Depression Rating Scale, 1979), certos itens do questionnário EPDS (Edinburgh postnatal scale depression, J. Cox, 1987) assim como certos itens do autoquestionário de Kennerley (1987). A avaliação das interações se baseia em uma escala de interação.

A ansiedade das mães cuja criança obteve um resultado positivo no teste de triagem não evolui entre a primeira e a segunda entrevista, seja ter sido o resultado unilateral $(\mathrm{t}(38)=1,83$; $\mathrm{n} . \mathrm{s})$ ou bilateral $(\mathrm{t}(38)=0,767 ; \mathrm{n} . \mathrm{s})$. Ocorre o mesmo para o estado psíquico das mães, que permanece está- vel entre as duas entrevistas. (Resultado unilateral: $\mathrm{t}(38)=1,019$, n.s; Resultado bilateral: $\mathrm{t}(38)$ $=0,767$, n.s). As interações permanecem com uma boa qualidade, e isto para todos os grupos considerados. Não se observam mais diferenças entre os grupos segundo o resultado do teste, na segunda entrevista.

\section{DISCUSSÃO}

O presente estudo tinha por objetivo avaliar os efeitos secundários de uma triagem sistemática da surdez neonatal. Os resultados se distinguem claramente dos estudos precedentes que concluíam haver uma ausência de ansiedade parental ligada à realização do teste de triagem 89. Com efeito, se bem que o teste em si mesmo não pareça ter consequências sobre o estado psicológico da mãe, um resultado positivo neste último acarreta um aumento importante da ansiedade materna que altera o seu estado psicológico geral, e tanto mais quanto o teste de triagem se revela positivo nas duas Grelhas. Apesar da alta ansiedade levantada nas mães, as interações observadas entre os dois parceiros permanecem de boa qualidade. Contudo, as mães cuja criança obteve um resultado positivo bilateral no teste de triagem apresentam menos confiança na maneira como elas gerem a interação com o seu recémnascido do que aquelas cujas crianças obtiveram um resultado negativo no mesmo teste.
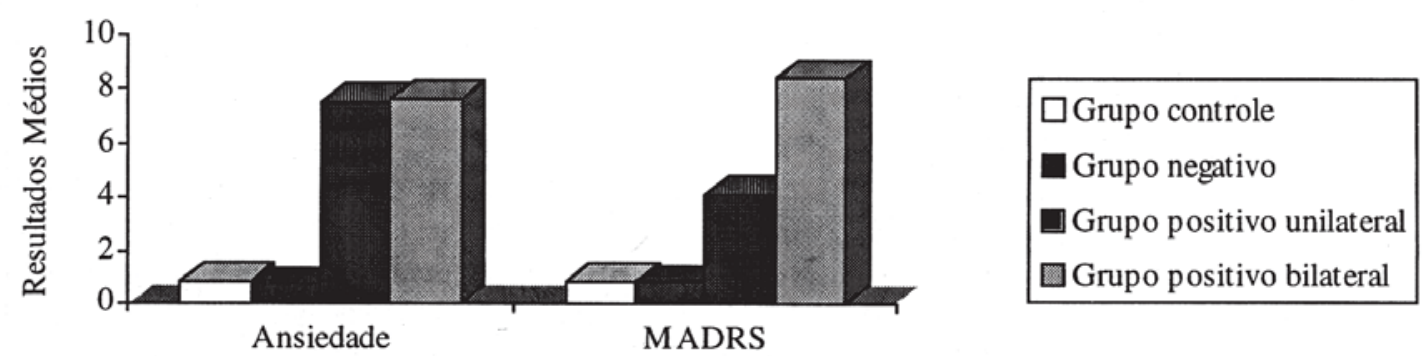

Gráfico 1: Avaliação do nível de ansiedade materna segundo o resultado do teste de triagem

Se bem que persistente no decorrer das semanas seguintes, a ansiedade materna, suscitada por um resultado positivo no teste de triagem, não aumenta e o estado psicológico das mães não se agrava. O nível de interação permanece estável entre as duas entrevistas.

Contudo, esses resultados não nos permitem avaliar a influência, em um período de tempo mais longo, de um resultado positivo no teste de triagem no estado psicológico da mãe assim como na realização das interações necessárias para a construção dos primeiros elos do apego. Dados retirados de pesquisas realizadas em enquadramento de um nascimento prematuro perm t rarn identificar, em certos pais, a realização, vários meses mais tarde, de um desengajamento emocional em relação à sua criança ou, de modo inverso, de um superenvolvimento preocupado nos cuidados, enquanto nada deixava prever a emergência de tal comportamento nas semanas que se seguiram à saída da criança do serviço de pediatria $1^{10-17}$. 
Eis porque é necessário realizar um acompanhamento psicológico imediato dos pais quando a sua criança apresenta um resultado positivo no teste de triagem da surdez neonatal permanente afim de evitar a mobilização de processos psicológicos que têm o risco de afetar de maneira mais ou menos irreversível o desenvolvimento da criança e das relações que ela manterá com os seus pais.

\begin{abstract}
The purpose of lhe present study is to assess lhe psychological impact of neonatal hearing screening on parental altitudes. Informing lhe parents about potential deafness in the neonatal period can produce excessivo parental anxiety and dysfunctional parent-child interactions. The results show high levei of parental anxiety, but lhe parent-child interaction is normal.
\end{abstract}

Key words: deafness. neonatal hearing screening. parental anxiety. neonatal interaction.

\section{REFERÊNCIAS}

1 Agence Nationale d'Accréditation et d'Evaluation dc la Santé (FR). Evaluation clinique et économique du dépistage néonatal de la surdité permanente par les otoémissions acoustiques provoquées. Paris:RapportJuin; 1999.

2 Appuzo ML, Yoshinaya-Itano C. Early identification of infants with significant hearing loss and the Minnesota Child Develop-ment In ventory. Semin Hear 1995; 16(2): 124-37.

3 Robinshaw HM Early intervention for hearing impairment: differences in the timing of communicative and linguistic development. $\mathrm{Br} \mathrm{J}$ Audiol 1995; 29: 315-34.

4 Yoshinaya-ltano C, Sedey AL, Coulter DK, Mehl AL. Language of early and lateridentified children withhearingloss. Pediatrics 1998; 102: 1161-71.

5 Pajulo M, Savonlathti E, Sourander A, Piha J, Helenius H. Prenatal maternal representations: mothers at psychosocial risk. Infant Ment Health J 2001; 22(5): 529-44.

6 Barringer DG, Mauk GW, Jensen S, Woods Kershner N. Survey of parents' perceptions regarding hospital-based newborn screening. Audiol Today 1997; 9(1): 18-9.

7 Magnuson M, Hergils L. The parents' view on hearing screening in newborns. Feelings, thoughts and opinions on otoacustic emissions screening. Scand Audiol 1999; 28: 47-56.
8 Weichbold V, Phil MD, Welzl-Mueller K. Maternal concern about positive test results in universal newborn hearing sreening. Pediatrics 2001; 108(5): 1111-6.

9 Weichbold V, Welzl-Mueller K, Mussbacher E. The impact of information on maternal attitudes towards universal neonatal hearing screening. Br J Audiol 2001; 35: 59-66.

10. Laganière J, Tessier R, Nadeau I. Attachment dans le cadre de la prématurité: un lien médiatisé par les perceptions maternelles. Enfance 2003; 55(2): 101- 18.

11 Fava-Vizziello G, Calvo V, Simonelli A. The effects of preterm birth on parental representations and child development. [Communication at $17^{\circ}$ Congress World Association for Mental Health; 2000 Juil 26-30; Montréal, Canada].

12 Stuart A, Moretz M, Yang EY. An investigation of maternal stress after neonatal hearing screening. AmJAudiol 2000; 9(2):135-41.

Recebido em 08/03/2004

Traduzido em 12/03/2004

Aprovado em 05/05/2004 